\title{
CARACTERIZAÇÃO FÍSICO-QUÍMICA E ATIVIDADE ANTIOXIDANTE (in vitro) DE FRUTOS DO CERRADO PIAUIENSE ${ }^{1}$
}

\author{
MARINA SOUZA ROCHA ${ }^{2}$, RAIMUNDO WILANE DE FIGUEIREDO ${ }^{3}$, \\ MARCOS ANTÔNIO DA MOTA ARAÚJO ${ }^{4}$, REGILDA SARAIVA DOS REIS MOREIRA-ARAÚJO
}

RESUMO - Informações a respeito das características químicas e do valor nutritivo dos frutos do cerrado são ferramentas básicas para a avaliação do consumo e para a formulação de novos produtos. No entanto, poucos dados estão disponíveis na literatura especializada com relação à composição química destes frutos e sua aplicação tecnológica, ressaltando a necessidade de pesquisas científicas sobre o assunto. Realizou-se o presente estudo com o objetivo de caracterizar físico-quimicamente e determinar a atividade antioxidante in vitro, pelo método DPPH, da cagaita (Eugenia dysenterica), cajuzinho-do-cerrado (Anacardium humile), chichá (Sterculia striata Naud.), jatobá-do-cerrado (Hymenaea stigonocarpa Mart.) e macaúba (Acrocomia aculeata Mart.). Os frutos foram colhidos na EMBRAPA - MEIO NORTE - PI, e na Cidade de Corrente-PI. Analisaram-se as características físicas (peso, comprimento e diâmetro), químicas (umidade, cinzas, lipídeos, proteínas, carboidratos), Valor Energético Total (VET), conteúdo de compostos fenólicos totais, flavonoides, antocianinas, $\beta$-caroteno, licopeno, vitamina $\mathrm{C}$ e atividade antioxidante pelo método DPPH. As amostras foram analisadas em dois lotes, e cada análise, em triplicata. Para a análise dos dados, foi elaborado um banco de dados, utilizando-se do programa estatístico EPI INFO, versão 6.04b, e o programa EXCEL. Os resultados obtidos para os compostos bioativos estudados demonstraram que todos os frutos apresentaram quantidade elevada de vitamina C (mg/100g), destacando-se o cajuzinho-do-cerrado (500). Os frutos que apresentaram maior capacidade antioxidante, expressos em capacidade de reduzir em $50 \%$ a atividade do radical livre DPPH (EC50mg/L), foram cagaita (Eugenia dysenterica Dc.) e cajuzinho-do-cerrado (Anacardium humile). Concluiu-se, portanto, que os frutos pesquisados apresentaram bom valor nutritivo, um elevado conteúdo de compostos bioativos e demonstraram atividade antioxidante.

Termos para Indexação: frutos nativos, compostos bioativos, capacidade antioxidante (DPPH).

\section{PHYSICAL AND CHEMICAL CHARACTERIZATION AND ANTIOXIDANT ACTIVITY (in vitro) OF FRUIT OF THE PIAUI SAVANNA}

\begin{abstract}
Details about the chemical characterist composition and nutritional value of the cerrado fruits are basic tools for evaluating the consumption and formulation of new products. However, few data are available in the literature with regard to chemical composition of these fruits and their technological application, highlighting the need for scientific research on the subject. It was performed the present study aiming at characterizing the physical-chemical and determine the antioxidant activity in vitro by the DPPH method, the cagaita (Eugenia dysenterica) cajuzinho-do-cerrado (Anacardium humile), chichá (Sterculia striata Naud .), jatoba-do-cerrado (Hymenaeastigonocarpa Mart.) and macaúba (Acrocomiaaculeata Mart.). The fruits were harvested at EMBRAPA - MID NORTH - PI and Correntes City - PI. It was analyzed the physical characteristics (weight, length and diameter), chemical (moisture, ashes, lipids, proteins, carbohydrates), total energy value (TEV), the content of total phenolics, flavonoids, anthocyanins, $\beta$-carotene, lycopene, vitamin C and antioxidant activity by DPPH. The samples were analyzed in two batches and each in triplicate analysis. For data analysis, it was designed a database, using the statistical program EPI INFO, version 6.04b. The obtained results for the studied bioactive compounds showed that all the fruits expressed hight amount of vitamin C (mg/100g), highlighting the cajuzinho-do-cerrado (500). The fruits with the highest antioxidant capacity, expressed in ability to reduce by $50 \%$ the activity of free radical DPPH (EC50mg / L) were cagaita (Eugenia dysenterica Dc.) and cajuzinho-do-cerrado (Anacardium humile). The research concludes, therefore, that the fruits studied showed good nutritional value, a meaningful content of bioactive compounds and demonstrate antioxidant activity.
\end{abstract}

Index terms: native fruits, bioactive compounds, antioxidant capacity (DPPH).

\footnotetext{
'(Trabalho 029-13). Recebido em: 03-01-2013. Aceito para publicação em: 16-09-2013.

${ }^{2}$ Nutricionista. Mestrado no Prog. de Pós-Graduação em Alimentos e Nutrição- UFPI. Tersina-PI. E-mail:marinasrocha@yahoo.com.br ${ }^{3}$ Eng. Agrônomo.Doutorado na Faculdade de Ciências Farmacêuticas da USP. Depto de Tecnologia de Alimentos-UFC. Campus do Pici. Fortaleza-CE.E-mail: figueira@ufc.br

${ }^{4}$ Estatístico, E-mail: regmarjoao@hotmail.com

${ }^{5}$ Nutricionista. Doutorado na Faculdade de Ciências Farmacêuticas da USP. Profa. Associada. Depto. de Nutrição/Programa de Pós-Graduação em Alimentos e Nutrição/UFPI. Campus Universitário Ministro Petrônio Portella. Bloco 13. Teresina-PI. E-mail: regilda@ufpi.edu.br
} 


\section{INTRODUÇÃO}

O consumo de frutas e vegetais tem sido associado à menor incidência de mortalidade por diversas doenças crônicas não transmissíveis. A proteção que esses alimentos oferecem contra as enfermidades degenerativas, como câncer, doenças cardiovasculares e cerebrovasculares, está associada ao seu elevado conteúdo de constituintes químicos com propriedades importantes, como antioxidantes (vitaminas $\mathrm{C}$ e E, carotenoides e polifenóis) (HINNEBURG et al., 2006).

Informações a respeito das características físico-químicas e do valor nutritivo e funcional dos frutos do cerrado são ferramentas básicas para incentivar o consumo e a formulação de novos produtos, pois o conhecimento das características físicas, dos macronutrientes, dos micronutrientes e dos compostos antioxidantes existentes nesses frutos possibilitará uma melhor indicação de seu consumo e utilização na indústria alimentícia. No entanto, poucos dados estão disponíveis na literatura especializada com relação à composição química destes frutos e sua aplicação tecnológica, ressaltando a necessidade de pesquisas científicas sobre o assunto (SILVA et al., 2008).

Dentre as possibilidades atuais de utilização das fruteiras do cerrado destacam-se: o plantio em áreas de proteção ambiental; o enriquecimento da flora das áreas mais pobres; a recuperação de áreas desmatadas ou degradadas; a formação de pomares domésticos e comerciais; e o plantio em áreas de reflorestamento, parques e jardins, e em áreas acidentadas. Nesse sentido, muitos agricultores e chacareiros já estão implantando pomares de frutas nativas dos cerrados, e os viveiristas estão intensificando a produção de mudas (AGOSTINICOSTA et al., 2010).

Antioxidantes são compostos que atuam inibindo e/ou diminuindo os efeitos desencadeados pelos radicais livres ou das espécies reativas não radicais. Tais ações podem ser alcançadas por meio de diferentes mecanismos de ação: impedindo a formação dos radicais livres ou espécies não radicais (sistemas de prevenção), impedindo a ação desses (sistemas varredores) ou, ainda, favorecendo o reparo e a reconstituição das estruturas biológicas lesadas (sistemas de reparo). São importantes, no combate aos processos oxidativos, com menores danos ao DNA e às macromoléculas, amenizando assim os danos cumulativos que podem desencadear doenças como o câncer, cardiopatias e cataratas (BARBOSA et al., 2010; SANTOS et al., 2008).

Os antioxidantes são qualquer substância que, quando presentes em baixas concentrações, comparadas à de um substrato oxidável, retardam ou inibem significativamente a oxidação deste substrato. Entre as principais enzimas responsáveis pela defesa antioxidante do organismo, destacam-se a superóxido dismutase (SOD), a catalase (CAT) e a glutationa peroxidase (GPx), que constituem a primeira defesa endógena de neutralização das espécies reativas de oxigênio (EROS). Por meio delas, as células tentam manter baixas as quantidades do radical superóxido e de peróxidos de hidrogênio, evitando, assim, a formação do radical hidroxil. As defesas não enzimáticas são compostas principalmente por antioxidantes, como,por exemplo, a glutationa (GSH), a vitamina $\mathrm{A}$, a vitamina $\mathrm{C}$, a vitamina $\mathrm{E}$, o zinco e o selênio (CANNIATTIBRAZACA;COUTO, 2010).

De acordo com especialistas da área, a incidência e a progressão de doenças crônicas são, em parte, desencadeadas justamente pelo balanço inadequado desses componentes funcionais nos sistemas biológicos, dentre os quais podemos citar os compostos fenólicos, os carotenoides, os flavonoides, as antocianinas, os terpenos, a vitamina $\mathrm{C}$, dentre outros fitoquímicos comumente presentes em quase todos os frutos encontrados na fruticultura brasileira. Em quantidades satisfatórias, as substâncias presentes nos frutos podem ser capazes de proteger as células contra processos degenerativos, atuando na possível prevenção de infecções e doenças, proporcionando a sustentabilidade e a manutenção de metabolismos reparatórios e compensadores, como a função antioxidante (NEVES, 2012).

Realizou-se o presente estudo com o objetivo de caracterizar físico-quimicamente e determinar a atividade antioxidante in vitro, pelo método DPPH, da cagaita (Eugenia dysenterica), cajuzinho-docerrado (Anacardium humile), chichá (Sterculia striata Naud.), jatobá-do-cerrado (Hymenaea stigonocarpa Mart.) e macaúba (Acrocomia aculeata Mart.).

\section{MATERIAL E MÉTODOS}

\section{Material}

Os frutos analisados no presente estudo foram: cagaita (Eugenia dysenterica), cajuzinhodo-cerrado (Anacardium humile), chichá (Sterculia striata Naud.), jatobá-do-cerrado (Hymenaea stigonocarpa Mart.) e macaúba (Acrocomia aculeata Mart.). Os frutos foram colhidos na EMBRAPA MEIO NORTE - PI, localizada no Município de Teresina, Capital do Estado do Piauí, que se encontra 
em latitude $05^{\circ} 05^{\prime} 21^{\prime \prime}$ e longitude $42^{\circ} 48^{\prime} 07^{\prime \prime}$ e na cidade de Corrente-PI, localizada no extremo sul Piauiense, latitude $10^{\circ} 26^{\prime} 36^{\prime \prime}$ e longitude $45^{\circ} 09^{\prime} 34^{\prime \prime}$. Utilizou-se de toda a parte comestível do fruto (casca e polpa), onde os frutos inteiros ou processados foram armazenados em freezer a $-12^{\circ} \mathrm{C}$ até o momento da análise. Para a análise dos dados, elaborou-se um banco de dados, utilizando-se do programa estatístico EPI INFO, versão 6.04b e o programa EXCEL.

\section{Métodos}

Caracterização física dos frutos, análise físico-química, macronutrientes e valor energético

A massa média foi determinada utilizandose da massa de 10 frutos, para a caracterização da amostra, considerando-se as medidas de comprimento (diâmetro maior) e largura (diâmetro menor), determinadas com Paquímetro modelo 530-101, segundo Instituto Adolfo Lutz (2008). Umidade, cinzas, lipídeos, proteínas totais e carboidratos foram analisados segundo a AOAC (2007). O Valor Energético Total - VET em $\mathrm{kcal} / 100 \mathrm{~g}$ foi calculado segundo os valores de conversão de Atwater de 4 $\mathrm{kcal} / \mathrm{g}$ de proteínas, $4 \mathrm{kcal} / \mathrm{g}$ de carboidratos e 9 $\mathrm{kcal} / \mathrm{g}$ de lipídeos, conforme a seguinte fórmula: $\mathrm{VET}=\{4$ (proteínas + carboidratos) +9 (lipídeos) $\}$ (WATT; MERRILL, 1963).

\section{Obtenção e preparo dos extratos Extração do extrato etéreo}

Utilizou-se de $15 \mathrm{~g}$ da amostra in natura (polpa e casca) com $100 \mathrm{~mL}$ de éter etílico e submeteu-se à agitação por $1 \mathrm{~h}$, em agitador automático. Após a agitação, a solução foi filtrada e centrifugada a $4.000 \mathrm{rpm}$, por $10 \mathrm{~min}$, para separar o líquido do sólido, sendo o líquido reservado (extrato etéreo) em um recipiente fechado ao abrigo da luz, e o resíduo restante foi submetido à secagem para ser utilizado na extração do extrato alcoólico, segundo Jardini e Mancini Filho (2007), adaptado.

\section{Extração do extrato alcoólico}

Misturou-se o resíduo seco do extrato etéreo e $100 \mathrm{~mL}$ de álcool etílico e repetiu-se a mesma operação realizada na elaboração do extrato etéreo. Em seguida, reservou-se o líquido em um recipiente fechado, e o sólido foi submetido à secagem para ser utilizado na extração do extrato aquoso, segundo Jardini e Mancini Filho (2007), adaptado.

\section{Extração do extrato aquoso}

Agitou-se por $1 \mathrm{~h}$ o resíduo seco do extrato alcoólico com $100 \mathrm{~mL}$ de água e repetiu-se a mesma operação realizada na elaboração do extrato etílico.
Reservou-se o líquido em um recipiente fechado, e o sólido (resíduo), descartado. Colocou-se $1 \mathrm{~mL}$, em triplicata, de cada extrato obtido em cápsulas, previamente taradas, e o material foi levado à estufa, a $105^{\circ} \mathrm{C}$, por $1 \mathrm{~h}$. Em seguida, realizou-se o cálculo para a obtenção da concentração dos extratos, segundo Jardini e Mancini Filho (2007), adaptado.

\section{Determinação de compostos fenólicos totais}

A quantificação de compostos fenólicos totais foi realizada pelo método espectrofotométrico, utilizando o reagente de Folin-Denins. Para o cálculo do teor de compostos fenólicos totais, adicionaramse em um balão volumétrico de $10 \mathrm{~mL}, 0,5 \mathrm{~mL}$ de amostra, em triplicata, do extrato alcoólico e aquoso, $8 \mathrm{~mL}$ de água destilada e $0,5 \mathrm{~mL}$ do reagente FolinDenins. Em seguida, a solução foi homogeneizada e deixada em repouso por $3 \mathrm{~min}$, e após este tempo acrescentou-se $1 \mathrm{~mL}$ de solução saturada de carbonato de sódio anidro. A solução ficou em repouso por 1 $\mathrm{h}$ e $\log$ o após foram realizadas as leituras das absorbâncias em espectrofotômetro de marca Bel SPECTRO S02 VIS, a $720 \mathrm{~nm}$, ao abrigo da luz. Para o cálculo da quantidade de compostos fenólicos totais, foi utilizada a equação da reta da curva-padrão construída com o ácido gálico nas concentrações de $0 ; 10 ; 15 ; 20$ e $35 \mathrm{mg} / \mathrm{L}$, segundo Swain e Hillis (1959), adaptada por Lima et al. (2007).

\section{Determinação de flavonoides e antocianinas}

Para o preparo do extrato, misturou-se $0,5 \mathrm{~g}$ da amostra com $10 \mathrm{~mL}$ de solução de etanol $95 \%$ + ácido clorídrico $(\mathrm{HCl}) 1,5 \mathrm{~N}$ previamente preparada (85:15). Homogeneizou-se e, logo após, transferiuse o conteúdo sem filtrar para um balão volumétrico de $25 \mathrm{~mL}$ e completou-se o volume do mesmo, até o traço de referência, com etanol $95 \%+\mathrm{HCl} 1,5 \mathrm{~N}$ $(85: 15)$. Deixou-se a solução descansando por uma noite sob refrigeração. Filtrou-se o material, ao abrigo da luz, determinando-se a absorbância, logo em seguida, em espectrofotômetro digital, marca Shimadzu 02900, Serial № A 114547 / UV- 1.800, a $535 \mathrm{~nm}$. Realizou-se o cálculo do teor de antocianinas multiplicando a Absorbância x fator de diluição/98,2, obtido, dividindo-se a massa da amostra $(0,5 \mathrm{~g})$ pelo volume da solução necessária $(25 \mathrm{~mL})$. O resultado foi correlacionado para $1 \mathrm{~mL}$ (quantidade de $\mathrm{g}$ que tem em $1 \mathrm{~mL}$ da solução) e determinou-se a quantidade de $\mathrm{mL}$ em $100 \mathrm{~g}$. O resultado final foi expresso em mL / 100g, segundo Francis (1982).

Para análise de flavonoides, realizou-se o mesmo procedimento e aplicou-se a mesma fórmula (absorbância x fator de diluição/98,2) para cálculo, 
sendo que as leituras das absorbâncias foram realizadas a $374 \mathrm{~nm}$.

\section{Determinação de carotenoides}

Para a obtenção do extrato, pesaram-se $2 \mathrm{~g}$ da amostra e acrescentaram-se $20 \mathrm{~mL}$ da mistura acetona - hexano na proporção (4:6). Agitou-se por 10 min em agitador magnético, em seguida filtrou-se a solução. Com o filtrado, fez-se a leitura em espectrofotômetro digital, marca Shimadzu 02900, Serial No A 114547 / UV- 1.800, nos seguintes comprimentos de onda: 453; 505; 645 e $663 \mathrm{~nm}$, segundo Nagata e Yamashita (1992), adaptado.

Para o cálculo do teor de $\beta$ - caroteno (em mg / $100 \mathrm{~mL}$ ), utilizou-se a equação 1 :

$0,216 \mathrm{xA}_{663}-1,22 \times \mathrm{A}_{645}-0,304 \times \mathrm{A}_{505}+0,452 \times \mathrm{A}_{453}$ EQ. 1

Para o cálculo do teor de licopeno (em mg / $100 \mathrm{~mL}$ ), utilizou-se a equação 2 :

$0,0458 \times \mathrm{A}_{663}+0,204 \times \mathrm{A}_{645}+0,372 \times \mathrm{A}_{505}-0,0806 \mathrm{xA}_{453}$ EQ.2

Em que: $\mathrm{A}=$ absorbância de cada leitura realizada.

Os resultados foram multiplicados por 1.000 e expressos em $\mu \mathrm{g} / 100 \mathrm{~g}$ de amostra fresca.

\section{Determinação de Vitamina C}

A concentração de ácido ascórbico (Vitamina C): foi determinada pelo Método de Tillmans, que se baseia na redução do 2,6-diclofenol indofenolsódio (DCFI) pelo ácido ascórbico, segundo Instituto Adolfo Lutz (2008).

\section{Determinação da Atividade Antioxidante pelo método de captura de radicais DPPH}

Este método baseia-se na redução do radical [2,2-difenil-1-pricril-hidrazil (DPPH)], que ao fixar um $\mathrm{H}$ (removido do antioxidante em estudo), leva a uma diminuição da absorbância, permitindo calcular, após o estabelecimento do equilíbrio da reação, a quantidade de antioxidante consumida na redução de $50 \%$ do radical DPPH. A partir dos extratos alcoólico e aquoso, a análise foi realizada em triplicata, acrescentando-se 1,5 mL de DPPH e $0,5 \mathrm{~mL}$ de amostra para cada extrato, e realizandose a leitura da absorbância em espectrofotômetro de marca Bel SPECTRO S02 VIS a $517 \mathrm{~nm}$, ao abrigo da luz, após 30 min do início da reação. Foi realizado este procedimento de leitura com quatro diluições/ concentrações diferentes, para cada extrato, obtidas a partir da concentração do extrato inicial.

A queda na leitura da densidade óptica das amostras foi correlacionada com o controle, estabelecendo-se a porcentagem de descoloração do radical DPPH, conforme a equação 3 a seguir:

$$
\begin{array}{r}
\% \text { de proteção }=\left(\mathrm{Abs}_{\text {controle }}-\mathrm{Abs}_{\text {amostra }}\right) \times 100 / \mathrm{Abs}_{\text {controle }} \\
\mathrm{EQ} .3
\end{array}
$$

Após o cálculo do percentual de proteção, construiu-se um gráfico com os resultados obtidos, lançando-se os valores de concentração (em mg/L) no eixo $\mathrm{X}$, e as porcentagens de proteção, no eixo Y. Foi determinada a equação da reta utilizada para o cálculo do valor do $\mathrm{EC}_{50}$ (quantidade de amostra necessária para reduzir em $50 \%$ a concentração inicial do radical DPPH), segundo Lima et al. (2007), desenvolvida por Blois (1958).

\section{RESULTADOS E DISCUSSÃO}

O conhecimento das características físicas dos frutos é de grande importância, tanto para se saber a diversidade de tamanho e massa em cada espécie, como para se viabilizar a confecção de embalagens para armazenamento e comercialização, de modo que não ocorram danos em sua estrutura física e promova melhor visualização frente ao consumidor.

Os resultados da característica física dos frutos analisados corroboram Corrêa et al. (2008) para cajuí. Para os demais frutos, não se obtiveram dados na literatura pesquisada para comparação. Observou-se que o jatobá apresentou maiores valores de massa e largura entre as amostras analisadas (Tabela 1).

A composição centesimal dos frutos nativos das regiões brasileiras, há tempos, vem sendo pesquisada e ainda não se conseguiu analisar todos os frutos que estão disponíveis, restando ainda uma vasta variedade de frutos nativos para serem estudados. Lopes et al. (2012) já alertavam que pesquisas vinham demonstrando que as regiões tropicais e subtropicais necessitavam de programas urgentes para estabelecer e processar fontes nativas de nutrientes e que essas medidas seriam satisfatórias para melhorar a dieta da população.

Dos frutos do Cerrado Piauiense analisados, verifica-se, na Tabela 02, que a cagaita mostrou um elevado teor de umidade $(90,0 \pm 8,4)$ e baixo valor calórico $(36,6 \pm 7,2)$; para o chichá, foram obtidos baixo teor de umidade $(8,4 \pm 1,5)$, elevados teores de lipídeos $(23,7 \pm 3,8)$ e calorias $(472,1 \pm 22,9)$, e comparando-se com os demais frutos, foi o que 
apresentou o maior valor calórico. O cajuzinhodo-cerrado demonstrou um elevado conteúdo de umidade $(82,6 \pm 9,7)$, baixo teor lipídico $(0,3 \pm 0,0)$ e valor calórico de $69,9 \pm 9,8$. Silva et al. (2008) observaram valores semelhantes para umidade $(86,57$ $\pm 0,11)$ e lipídeos $(0,63 \pm 0,05)$, e discordante para o valor calórico $(38,27)$. Dado que pode ser justificado devido à diferença de local de colheita, solo, clima e época de frutificação; o jatobá apresentou maior teor de cinzas $(5,0 \pm 0,8)$ dentre os frutos analisados e baixo teor de umidade $(12,0 \pm 2,1)$. Para macaúba, obteve-se teor de cinzas $(2,3 \pm 0,2)$, carboidratos $(36,4 \pm 5,2)$ e calorias $(296,9 \pm 12,9)$, ficando em terceiro lugar dentre os frutos analisados. Nestes teores, observou-se um elevado conteúdo de lipídeos $(16,6 \pm 3,2)$, ficando em segundo lugar dentre os frutos analisados. Comparando-se com os dados verificados por Silva et al. (2008), observaram-se valores equivalentes para cinzas $(1,78 \pm 0,02)$, lipídeos $(14,93 \pm 0,21)$, carboidratos $(35,06 \pm 0,40)$ e calorias $(285,65)$.

A Figura 01 mostra os resultados para compostos fenólicos totais (mg GAE / $100 \mathrm{~g}$ do fruto) em extrato alcoólico e aquoso. Observou-se que o fruto que obteve maior destaque na extração alcoólica foi o chichá $(85,37 \pm 7,77)$, seguido da macaúba $(60,85 \pm 11,15)$, cajuzinho-do-cerrado $(51,15 \pm 0,00)$ e jatobá $(34,10 \pm 2,13)$. Com relação à extração aquosa, o fruto que mais se destacou foi o chichá $(281,31 \pm 7,05)$. O que apresentou menor valor foi o cajuzinho-do-cerrado $(11,81 \pm 2,67)$. Estes resultados expressam a capacidade de extração do álcool etílico frente aos compostos fenólicos existentes nos frutos analisados.

Segundo Marques et al. (2012), analisando o fruto da castanhola, empregando a mesma metodologia de extração utilizada no presente estudo, foi mostrado que a água foi o melhor solvente para a extração dos compostos fenólicos da castanhola.

Observou-se que os frutos do cerrado demonstraram teor de compostos fenólicos totais comparáveis com os resultados obtidos por Faller e Fialho (2009), mg GAE/mL, na determinação de polifenóis em frutas no Brasil: o abacaxi apresentou $85,1( \pm 5,8)$, a banana $215,7( \pm 3,5)$, a laranja 114,6 $( \pm 1,3)$, o mamão $15,3( \pm 0,3)$, a manga $110,5( \pm 9,6)$ e a tangerina $134,1( \pm 6,5)$. Enquanto Vieira et al. (2011), em polpas congeladas de frutas, detectaram que a acerola apresentou $835,25 \pm 32,44 \mathrm{mg} / 100 \mathrm{~g}$ de fenólicos totais para o extrato aquoso e 449,63 $\pm 10,24 \mathrm{mg} / 100 \mathrm{~g}$ para o extrato hidroalcoólico; a polpa de caju apresentou os valores de 201,61 \pm 19,15 e $165,07 \pm 4,10 \mathrm{mg} / 100 \mathrm{~g}$, para os extratos aquoso e hidroalcoólico, respectivamente; a polpa de goiaba $104,76 \pm 4,39$ e $20,2 \pm 1,95$; as polpas de bacuri com 10,35 $\pm 0,42$ e 7,23 $\pm 0,08 \mathrm{mg} / 100$ g, e de tamarindo com $23,57 \pm 0,36$ e 23,35 $\pm 0,21$ $\mathrm{mg} / 100 \mathrm{~g}$ para os extratos aquoso e hidroalcoólico, respectivamente. $\mathrm{O}$ fato de os resultados das polpas congeladas demonstrarem, em alguns frutos, teores mais elevados do que os verificados no presente estudo, pode ser explicado pela maior concentração do fruto na polpa e pelos diferentes tipos de extração.

$\mathrm{O}$ fruto que apresentou maior teor em $\mathrm{mg} /$

$100 \mathrm{~g}$ para antocianinas e flavonoides, foi o jatobá, com $0,88 \pm 0,4$ e $19,64 \pm 1,5$, respectivamente (Tabela 03). Segundo Rocha et al. (2011), os compostos fenólicos geralmente estão associados ao mecanismo de adaptação e resistência da planta ao meio ambiente, podendo influenciar no sabor, nas características tecnológicas, como escurecimento ou precipitação durante o processamento, assim como no potencial nutritivo e funcional destas frutas.

Destacou-se, em todos os frutos, um elevado teor de Vitamina C, em comparação com a Recomendação Diária de 45 mg (BRASIL, 2005) (Tabela 03). Comparando-se o resultado com dados obtidos por Canniatti-Brazaca e Couto (2010), observou-se que os teores de ácido ascórbico expressos em vitamina $\mathrm{C}$ existentes nos frutos do Cerrado Piauiense foram superiores à maioria das frutas cítricas, com resultados expressos em ácido ascórbico (mg.100 mL-1 suco): Tangerina poncã $32,47 \pm 1,791$; Tangerina murcote $21,47 \pm 1,11$; Laranja-pera $62,50 \pm 0,96$; Laranja-lima $64,58 \pm$ 0,46; Laranja-natal 84,03 $\pm 3,18$; Laranja-valência $78,47 \pm 1,20$; Laranja-baia $80,03 \pm 1,03$.

Todos os frutos analisados (Tabela 03), com exceção do chichá, apresentaram valores de $\beta$-caroteno em $\mu \mathrm{g} / 100 \mathrm{~g}$ do fruto, com destaque para a cagaita $(201,23 \pm 25,1)$. Quanto ao licopeno, a maioria dos frutos não o apresentou. Apenas o chichá e o jatobá apresentaram conteúdo de licopeno de $119,78 \pm 9,78$ e $9,96 \pm 1,23$, respectivamente. Rodrigues-Amaia et al. (2007), em estudo realizado sobre o teor de carotenoides em frutas e outros vegetais, chamaram a atenção que sobre frutos da mesma cultivar, pois quando produzidos nos estados do Nordeste, apresentaram teores de carotenoides expressivamente mais elevados do que aqueles produzidos no clima temperado do Estado de São Paulo e relataram que isso comprova a estimulação da biossíntese de carotenoides com exposição ao sol;no entanto, podem promover também a fotodegradação.

Os resultados obtidos para capacidade antioxidante, expressos na capacidade de reduzir em $50 \%$ o radical DPPH (EC50 mg / L), de frutos do Cerrado, extraídos com extrato alcoólico e aquoso, 
demonstrados na Figura 02, apontam um elevado poder antioxidante para os frutos que apresentaram capacidade de redução em $50 \%$ do radical livre DPPH com a menor concentração. Cada fruto teve um comportamento diferenciado no que diz respeito ao tipo de extrato, demonstrando precisar de maior ou menor concentração para reduzir em 50\% a atividade do radical livre (DPPH).

Atualmente, é difícil comparar, baseando-se na literatura científica, a atividade antioxidante de diferentes amostras, devido aos autores utilizarem diferentes diluições das amostras para a realização da análise, diferentes tipos de solventes e métodos para a obtenção dos resultados, visto que cada amostra apresenta um poder antioxidante e comporta-se de forma diferente em cada tipo de análise. Contudo, os resultados obtidos apontam que os frutos que apresentaram a melhor capacidade de reduzir o radical livre em 50\% foram a cagaita com 430,92 mg / L (extrato alcoólico) e 970,27 mg / L (extrato aquoso) e o cajuzinho-do-cerrado com $881,7 \mathrm{mg}$ / L (extrato alcoólico) e 1050,17 mg / L (extrato aquoso),podendo tal capacidade ser se atribuída ao teor de $\beta$-caroteno e vitamina $\mathrm{C}$ desses frutos. Comparando-se os resultados obtidos no presente estudo, com os resultados obtidos por Rufino et al. (2010), que observaram capacidade antioxidante em reduzir $50 \%$ de grama do radical por grama do fruto (EC50g/g DPPH), observou-se que o açaí necessitou de $598 \pm 164$; a acerola de 49,2 $\pm 2,5$, o cajá de 1064 \pm 162 e o caju de $906 \pm 78,2$.

Interessante ressaltar, segundo Oliveira et al. (2011), que a atividade antioxidante in vivo de frutas tropicais deve ser compreendida como o resultado da soma de vários compostos bioativos, o que reforça a importância da variedade na composição de refeições, e expõe as limitações de proposições que valorizam, mais exclusiva e isoladamente, um ou outro componente específico da alimentação.

TABELA 1- Caracterização física de frutos do Cerrado Piauiense.

\begin{tabular}{cccc}
\hline \hline FRUTOS & PESO $(\mathbf{g}) *$ & $\begin{array}{c}\text { COMPRIMENTO } \\
(\mathbf{m m})\end{array}$ & $\begin{array}{c}\text { LARGURA } \\
(\mathbf{m m})\end{array}$ \\
\hline \hline Cagaita & $9,3 \pm 2,8$ & $23,1 \pm 5,2$ & $271 \pm 5,8$ \\
Chichá & $1,7 \pm 0,1$ & $18,67 \pm 1,8$ & $12,5 \pm 3,8$ \\
Cajuí & $7,9 \pm 1,3$ & $34,2 \pm 7,9$ & $21,8 \pm 4,2$ \\
Jatobá & $57,5 \pm 6,7$ & $94,25 \pm 9,5$ & $37,2 \pm 3,4$ \\
Macaúba & $21,5 \pm 2,8$ & $35,2 \pm 8,5$ & $34,1 \pm 4,1$ \\
\hline
\end{tabular}

*Valores apresentados em Média e \pm Desvio- padrão: três repetições/amostra

TABELA 2- Composição centesimal (g/100 gramas do fruto) e Valor Energético Total (VET), da amostra úmida de frutos do Cerrado Piauiense.

\begin{tabular}{|c|c|c|c|c|c|c|}
\hline FRUTOS & $\begin{array}{l}\text { Umidade* }^{*} \\
\text { (g) }\end{array}$ & $\begin{array}{c}\text { Cinzas } \\
(\mathrm{g})\end{array}$ & $\begin{array}{l}\text { Lipídeos } \\
\text { (g) }\end{array}$ & $\begin{array}{l}\text { Proteína } \\
\text { (g) }\end{array}$ & $\begin{array}{c}\text { Carboidrato } \\
\text { (g) }\end{array}$ & $\begin{array}{l}\text { VET } \\
\text { (kcal) }\end{array}$ \\
\hline Cagaita & $90,9 \pm 8,4$ & $0,3 \pm 0,1$ & $0,3 \pm 0,1$ & $2,5 \pm 0,2$ & $5,9 \pm 1,7$ & $36,6 \pm 7,2$ \\
\hline Chichá & $8,4 \pm 1,5$ & $3,1 \pm 0,2$ & $23,7 \pm 3,8$ & $13,8 \pm$ & $50,9 \pm 9,8$ & $472,1 \pm 22,9$ \\
\hline Cajuí & $82,6 \pm 9,7$ & $0,3 \pm 0,0$ & $0,3 \pm 0,0$ & & $15,7 \pm 4,9$ & $69,9 \pm 9,8$ \\
\hline Jatobá & $12,0 \pm 2,1$ & $5,0 \pm 0,8$ & $1,36 \pm 0,2$ & $1,1 \pm 0,1$ & $79,8 \pm 17,3$ & $337,87 \pm 6,4$ \\
\hline Macaúba & $44,1 \pm 6,9$ & $2,3 \pm 0,2$ & $16,6 \pm 3,2$ & $\begin{array}{l}1,1 \pm 0,3 \\
0,6 \pm 0,1\end{array}$ & $36,4 \pm 5,2$ & $296,9 \pm 12,9$ \\
\hline
\end{tabular}

*Valores apresentados em Média e \pm Desvio-padrão: três repetições/amostra 
TABELA 3 - Compostos bioativos em frutos do cerrado Piauiense.

\begin{tabular}{|c|c|c|c|c|c|}
\hline FRUTOS & $\begin{array}{l}\text { VITAMINA C* } \\
\mathrm{mg} / 100 \mathrm{~g}\end{array}$ & $\begin{array}{c}\text { FLAVONOIDES } \\
\mathrm{mg} / 100 \mathrm{~g}\end{array}$ & $\begin{array}{c}\text { ANTOCIANINAS } \\
\mathrm{mg} / 100 \mathrm{~g}\end{array}$ & $\begin{array}{c}\text { LICOPENO } \\
\mu \mathrm{g} / \mathbf{1 0 0 g}\end{array}$ & $\begin{array}{c}\beta \text {-CAROTENO } \\
\mu \mathrm{g} / 100 \mathrm{~g}\end{array}$ \\
\hline Cagaita & $126,3 \pm 45,8$ & $9,51 \pm 0,4$ & $0,38 \pm 0,8$ & nd** & $201,23 \pm 25,1$ \\
\hline Chichá & $89,3 \pm 9,8$ & $2,81 \pm 0,6$ & $0,88 \pm 0,4$ & $119,78 \pm 9,78$ & nd** \\
\hline Cajuí & $500,0 \pm 89,7$ & $3,12 \pm 0,7$ & $0,22 \pm 0,7$ & $\mathrm{nd}^{* *}$ & $136,13 \pm 18,3$ \\
\hline Jatobá & $330,4 \pm 61,5$ & $19,64 \pm 1,5$ & $2,12 \pm 0,7$ & $9,96 \pm 1,23$ & $110,68 \pm 11,9$ \\
\hline Macaúba & $185,1 \pm 14,8$ & $4,56 \pm 0,3$ & $0,57 \pm 0,9$ & nd** & $132,65 \pm 17,2$ \\
\hline
\end{tabular}

*Valores apresentados em Média e \pm Desvio-padrão: três repetições/amostra. **Não detectado.

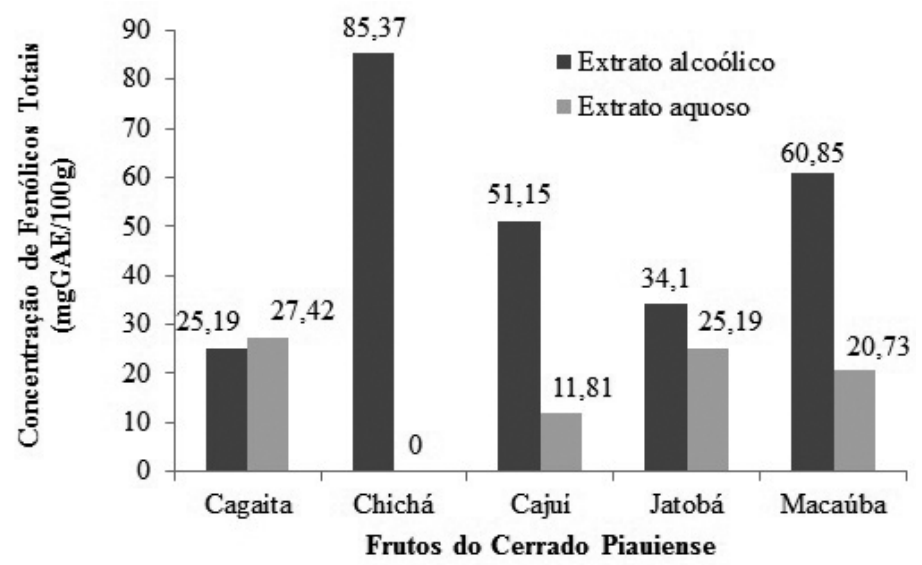

FIGURA 1- Teor de compostos fenólicos totais (mgGAE/100g), do extrato alcoólico e aquoso, da cagaita, chichá, cajuí, jatobá e macaúba.

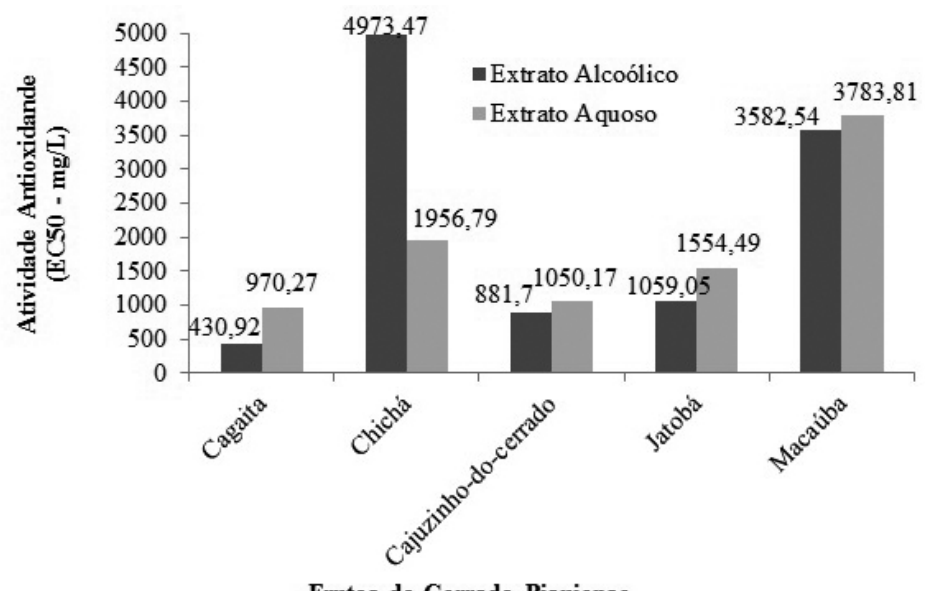

Frutos do Cerrado Piauiense

FIGURA 2 - Atividade antioxidante expressas em $\mathrm{EC}_{50}$, dos extratos alcoólico e aquoso, pelo método DPPH, da cagaita, chichá, cajuí, jatobá e macaúba. 


\section{CONCLUSÃO}

Os frutos do Cerrado analisados demonstram possuir características físico-químicas, nutritivas e funcionais que possibilitam sua utilização na indústria de alimentos. Os frutos que se destacam pelo conteúdo de fenólicos totais, para o extrato alcoólico, são o chichá, a macaúba e o cajuí e, para o extrato aquoso, a cagaita e o jatobá-do-cerrado. Quanto aos flavonoides e antocianinas, o jatobá-do-cerrado apresenta o maior conteúdo. Os frutos que apresentam maior teor de $\beta$-caroteno foram a cagaita e o cajuzinho-do-cerrado; de licopeno são o chichá e o jatobá-do-cerrado; e de vitamina $\mathrm{C}$ o cajuí. A cagaita e o cajuzinho-do-cerrado são os frutos que demonstram maior poder antioxidante in vitro, tanto no extrato aquoso quanto no alcoólico.

\section{AGRADECIMENTOS}

Ao CNPq, pelo financiamento concedido por meio do Edital Universal, processo $n^{\circ} 481.333 / 2007$ Edital MCT/CNPq 15/2007. À CAPES, pela bolsa de mestrado concedida.

\section{REFERÊNCIAS}

AGOSTINI-COSTA, T. S.; SILVA, D. B.; VIEIRA, R. F.; SANO, S. M.; FERREIRA, F. R. Espécies de maior relevância para a região Centro-Oeste. In: VIEIRA, R. F.; AGOSTINI-COSTA, T. S.; SILVA, D. B.; SANO, S.; FERREIRA, F. R. Frutas nativas da região Centro-Oeste. Brasília: Embrapa, 2010. p. 15-30.

AOAC - Association of Official Analytical Chemists International. Official Methods of Analysis of AOAC International. $18^{\text {th }}$ ed. Gaitherburg: AOAC International, 2007.

BARBOSA, K. B. F.; COSTA, N. M. B.; ALFENAS, R. de C. G.; DE PAULA, S. O.; MINIM, V. P. R.; BRESSAN, J. Estresse oxidativo: conceito, implicações e fatores modulatórios. Revista de Nutrição, Campinas, n. 23, v. 4, p. 629-643, 2010.

BLOIS, M.S. Antioxidant determinations by the use of a stable free radical. Nature, London, n.181, v.1, p. 199-200, 1958.
BRASIL. Ministério da Saúde. Agência Nacional de Vigilância Sanitária. Resolução RDC nº 269, de 22 de setembro de 2005. Regulamento técnico sobre a ingestão diária recomendada (IDR) de proteína, vitaminas e minerais. Brasília, 2005.

CANNIATTI-BRAZACA, S. G.; COUTO, M.A. L. Quantificação de vitamina $\mathrm{C}$ e capacidade antioxidante de variedades cítricas. Ciências e Tecnologia de Alimentos, Campinas, v. 30, p. 1519, 2010.

CORRÊA, G. de C.; NAVES, R. V.; ROCHA, M R.; CHAVES, L. J.; BORGES, J. D. Determinações físicas em frutos e sementes de baru (Dipteryx alata vog.), cajuzinho (Anacardium othonianum rizz.) e pequi (Caryocar brasiliense camb.), visando ao melhoramento genético. Bioscience Journal, Uberlândia, v. 24, n. 4, p. 42-47, 2008.

COUTO, M. A. L.; CANNIATTI-BRAZACA, S. G. Quantificação de vitamina $C$ e capacidade antioxidante de variedades cítricas. Ciência e Tecnologia dos Alimentos, Campinas, n. 30. v.1, p. 15-19, 2010.

FALLER, A. L. K.; FIALHO, E. Disponibilidade de polifenóis em frutas e hortaliças consumidas no Brasil. Revista de Saúde Pública, São Paulo, v. 43, n. 2, p. 211-218, 2009.

FRANCIS, F.J. Analysis of anthocyanins. In: MARKAKIS, P. (Ed.). Anthocyanins as food colors. New York: Academic Press, 1982. p.181-207.

HINNEBURG, I.; DAMIEN,H.J.; RAIMO, H Antioxidant activities of extracts from selected culinary herbs and spices. Food Chemistry, London, v. 97, n. 1 , p. $122-129,2006$.

INSTITUTO ADOLFO LUTZ. Métodos físicosquímicos para análise de alimentos. 4.ed. Brasília: Ministério da Saúde, Agência Nacional de Vigilância Sanitária, 2008.

JARDINI, F. A.; MANCINI FILHO, J. Avaliação da atividade antioxidante em diferentes extratos da polpa e sementes da romã (Punica granatum, L.). Revista Brasileira de Ciências Farmacêuticas, São Paulo, v. 43, n. 1, p. 137-147, 2007. 
LIMA, A.; SILVA, A. M. de O. E.; TRINDADE, R.A.; TORRES, R. P.; MANCINI-FILHO, J. Composição química e compostos bioativos presentes na polpa e na amêndoa do pequi (Caryocar brasiliense, Camb.). Revista Brasileira de Fruticultura, Jaboticabal, v. 29, n. 3. p 155-159. 2007.

LOPES, R. M.; SILVA, J. P. da; VIEIRA, R. F.; SILVA, D. B. da;GOMES, I. da S.; AGOSTINICOSTA, T. da S. Composição de ácidos graxos em polpa de frutas nativas do cerrado. Revista Brasileira de Fruticultura, Jaboticabal, v. 34, n.2, p. $635-640,2012$.

MARQUES, M. R.; PAZ, D. D.; BATISTA, L. P. R.; BARBOSA, C. de O.; ARAÚJO, M. A. M.; MOREIRA-ARAÚJO, R. S. dos R. An in vitro analysis of the total phenolic content, antioxidant power, physical, physicochemical, and chemical composition of Terminalia Catappa Linn fruits. Ciência e Tecnologia de Alimentos, Campinas, v. 32, n. 1, p. 209-213, 2012.

NAGATA, M.; YAMASHITA, I. Simple method for simultaneous determination of chlorophyll and carotenoids in tomato fruit. Nippon Shokuhin Kogyo Gakkaishi, Tokyo, v. 39, n. 10, p. 925-928, 1992.

NEVES, L. C. Frutos - O remédio do futuro! Revista Brasileira de Fruticultura, Jaboticabal, v. 34 n. 4, p. i, 2012.

OLIVEIRA, D. da S.; AQUINO, P. P.; RIBEIRO, S. M. R.; PROENÇA, R. P. da C.; PINHEIROSANT'ANA, H. M. Vitamina C, carotenoides, fenólicos totais e atividade antioxidante de goiaba, manga e mamão procedentes da Ceasa do Estado de Minas Gerais. Acta Scientiarum. Health Sciences, Maringá, v. 33, n. 1, p. 89-98, 2011.

ROCHA, W. S.; LOPES, R. M.; SILVA, D. B. da; VIEIRA, R. F.; SILVA, J. P. da; AGOSTINI-COSTA, T. da S. Compostos fenólicos totais e taninos condensados em frutas nativas do cerrado. Revista Brasileira de Fruticultura, Jaboticabal, v. 33, n. 4, p. 1215-1221, 2011.
RODRIGUEZ-AMAYA, D. B.; PORCU, M. M.; AZEVEDO-MELEIRO, C. H. Varation in the Carotenoid Composition of Fruits and Vegetables along the Food Chain. Acta Horticulturae, The Hague, v. 744, n.1, p.387-394, 2007.

RUFINO, M. do S. M.; ALVES, R. E.; BRITO, E. S. de; PÉREZ-JIMÉNEZ, J.; SAURA-CALIXTO, F.; MANCINI-FILHO, J. Bioactive compounds and antioxidant capacities of 18 non-traditional tropical fruits from Brazil. Food Chemistry, London, v. 121, n. 4, p. 996-1.002, 2010.

SANTOS, G. M. dos; MAIA, G. A.; SOUSA, P. H. M. de; COSTA, J. M. C. da C.; FIGUEIREDO, R. W. de; PRADO, G. M. do. Correlação entre atividade antioxidante e compostos bioativos de polpas comerciais de açaí (Euterpe oleracea Mart). Archivos Latinoamericanos de Nutricion, Caracas, v. 58, n. 2, p. 187-192, 2008.

SILVA, M. R.; LACERDA, D. B. C. L.; SANTOS, G. G.; MARTINS, D. M. de O. Caracterização química de frutos nativos do cerrado. Ciência Rural, Santa Maria, v.38, n.6, p. 1790-1793, 2008.

SWAIN, T.; HILLIS, W.E. The phenolic constituents of Prunus domesticaI. The quantitative analysis of phenolic constituents. Journal of the Science of Food and Agriculture, London, v.10, p. 63-68, 1959.

VIEIRA, L. M.; SOUSA, M. S. B.; MANCINIFILHO, J.; LIMA, A. de. Fenólicos totais e capacidade antioxidante. Revista Brasileira de Fruticultura, Jaboticabal, v. 33, n. 3, p. 888-897, 2011.

WATT, B.; MERRILL, A. L. Composition of foods: raw, processed, prepared. Washington: Consume and Food Economics Research. Divison/Agricultural Service, 1963. (Agriculture Handbook, 8) 